\title{
Relationship of a Variant in the NTRK1 Gene to White Matter Microstructure in Young Adults
}

\author{
Meredith N. Braskie, ${ }^{1}$ Neda Jahanshad, ${ }^{1,2}$ Jason L. Stein, ${ }^{1}$ Marina Barysheva, ${ }^{1}$ Kori Johnson, ${ }^{4,6}$ Katie L. McMahon, ${ }^{4}$ \\ Greig I. de Zubicaray, ${ }^{5}$ Nicholas G. Martin, ${ }^{6}$ Margaret J. Wright, ${ }^{6}$ John M. Ringman, ${ }^{3}$ Arthur W. Toga, ${ }^{1}$ \\ and Paul M. Thompson ${ }^{1}$ \\ ${ }^{1}$ Laboratory of Neuro Imaging, Department of Neurology and 2Medical Imaging Informatics Group, Department of Radiology, University of California, Los \\ Angeles (UCLA) School of Medicine, Los Angeles, California 90095, ${ }^{3}$ Mary S. Easton Center for Alzheimer's Disease Research, Department of Neurology, \\ UCLA, Los Angeles, California 90095, ${ }^{4}$ Centre for Advanced Imaging and ${ }^{5}$ School of Psychology, University of Queensland, Brisbane, Queensland 4072, \\ Australia, and ${ }^{6}$ Queensland Institute of Medical Research, Brisbane, Queensland 4029, Australia
}

The NTRK1 gene (also known as TRKA) encodes a high-affinity receptor for NGF, a neurotrophin involved in nervous system development and myelination. NTRK1 has been implicated in neurological function via links between the T allele at rs6336 (NTRK1-T) and schizophrenia risk. A variant in the neurotrophin gene, BDNF, was previously associated with white matter integrity in young adults, highlighting the importance of neurotrophins to white matter development. We hypothesized that NTRK1-T would relate to lower fractional anisotropy in healthy adults.

We scanned 391 healthy adult human twins and their siblings (mean age: $23.6 \pm 2.2$ years; 31 NTRK1-T carriers, 360 non-carriers) using 105-gradient diffusion tensor imaging at 4 tesla. We evaluated in brain white matter how NTRK1-T and NTRK1 rs4661063 allele A (rs4661063-A, which is in moderate linkage disequilibrium with rs6336) related to voxelwise fractional anisotropy-a common diffusion tensor imaging measure of white matter microstructure. We used mixed-model regression to control for family relatedness, age, and sex. The sample was split in half to test reproducibility of results. The false discovery rate method corrected for voxelwise multiple comparisons.

NTRK1-T and rs4661063-A correlated with lower white matter fractional anisotropy, independent of age and sex (multiple-comparisons corrected: false discovery rate critical $p=0.038$ for NTRK1-T and 0.013 for rs $4661063-\mathrm{A}$ ). In each half-sample, the NTRK1-T effect was replicated in the cingulum, corpus callosum, superior and inferior longitudinal fasciculi, inferior fronto-occipital fasciculus, superior corona radiata, and uncinate fasciculus. Our results suggest that NTRK1-T is important for developing white matter microstructure.

\section{Introduction}

The NTRK1 gene (also known as TRKA) encodes the neurotrophic tyrosine kinase, receptor, type 1: a high-affinity receptor for NGF. NTRK1 is implicated in guiding nervous system development (Maglott et al., 2005). Dysfunctions of neurotrophin signaling pathways, such as for $N G F$ and $B D N F$, may make

Received Nov. 4, 2011; revised Feb. 12, 2012; accepted March 5, 2012.

Author contributions: M.N.B., N.J., J.L.S., M.B., K.J., K.L.M., G.I.d.Z., N.G.M., M.J.W., J.M.R., A.W.T., and P.M.T. designed research; K.J., K.L.M., G.I.d.Z., N.G.M., and M.J.W. performed research; M.N.B., N.J., J.L.S., M.B., and P.M.T. analyzed data; M.N.B., N.J., J.L.S., K.L.M., G.I.d.Z., N.G.M., M.J.W., J.M.R., A.W.T., and P.M.T. wrote the paper.

This study was supported by the National Institute of Child Health and Human Development (Grant R01 HD050735), and the National Health and Medical Research Council (NHMRC 486682), Australia. Genotyping was supported by the NHMRC (Grant 389875). Additional support was provided by NIH R01 Grants AG040060, EB008432, EB008281, and EB007813. M.N.B. was funded in part by the NIH (Grant P50 AG-16570) and by the UCLA Easton Consortium for Biomarker and Drug Discovery in Alzheimer's Disease. N.J. was funded in part by the NIH-National Library of Medicine (T15 LM07356). J.L.S. was also funded by the ARCS Foundation and the NIMH (F31MH087061). G.I.d.Z. is supported by an Australian Research Council (ARC) Future Fellowship (FT0991634). We thank the twins and siblings for their participation. In Brisbane, we thank Marlene Grace and Ann Eldridge for twin recruitment, Aiman AI Najjar and other radiographers for scanning, Kerrie McAloney and Daniel Park for research support, and staff in the Molecular Epidemiology Laboratory for DNA sample processing and preparation.

The authors declare no competing financial interests.

Correspondence should be addressed to Dr. Paul Thompson, Laboratory of Neuro Imaging, Department of Neurology, UCLA School of Medicine, 635 Charles Young Drive South, Suite 225, Los Angeles, CA 90095-7334. E-mail: thompson@loni.ucla.edu.

DOI:10.1523/JNEUROSCI.5561-11.2012

Copyright $\odot 2012$ the authors $\quad 0270-6474 / 12 / 325964-09 \$ 15.00 / 0$ the brain more vulnerable to schizophrenia (Shoval and Weizman, 2005). Our research group previously found that the BDNF Val allele at Val66Met (rs6265) is associated with differences in white matter microstructure (Chiang et al., 2011), making other neurotrophins likewise attractive targets for the study of white matter development. Additionally, the proteins NGF and NTRK1 have been implicated in regulating CNS myelination (Chan et al., 2004; Lee et al., 2007). Here we evaluated how white matter microstructure relates to the $\mathrm{T}$ allele of a common variant in the NTRK1 gene at rs6336 (NTRK1-T). We also examined how white matter microstructure related to a variant (rs4661063) whose alleles were moderately correlated (in linkage disequilibrium) with rs6336 since similar but weaker effects in related variants add support to the effect of interest. We tested the specificity of our results by examining whether top variants thought to contribute to schizophrenia and multiple sclerosis, two diseases known to affect white matter (Peters et al., 2010; Ramli et al., 2010; Thomason and Thompson, 2011), were associated with white matter microstructure in our sample.

The single-nucleotide polymorphism rs6336 within the NTRK1 gene is a nonsynonymous change whose minor allele (T) has been associated with an increased risk of developing schizophrenia relative to the C allele (van Schijndel et al., 2009), although those results were not consistent (Van Schijndel et al., 
2011). Approximately $8 \%$ of Caucasians carry the NTRK 1 -T variant (The International HapMap 3 Consortium, 2010), depending on the cohort examined (Van Schijndel et al., 2011). Our goal was to evaluate white matter microstructure, not schizophrenia risk, in this sample. However, the relationship of NTRK1-T to schizophrenia suggests that the variant affects the brain, increasing the likelihood that a relationship between the variant and brain structure will be detectable using MRI.

Fractional anisotropy (FA), an index derived from diffusion tensor imaging (DTI), measures the extent to which water diffusion is directionally constrained. Higher FA typically indicates greater brain fiber myelination or coherence [with some exceptions (Thomason and Thompson, 2011)]. We hypothesized that rs6336 allele T (NTRK1-T) would be associated with lower FA in healthy adults.

\section{Materials and Methods}

Subjects and genotype information. We acquired high-angular resolution DTI scans and genotypes for 468 right-handed healthy young adults of European ancestry recruited as part of a study to examine brain structure and function in Australian twins from the Queensland Twin IMaging (QTIM) study. All subjects already had genome-wide genotyping, although the results presented here are not part of a genome-wide analysis. From these 468 adults, we excluded 77 subjects for the following reasons: 8 were ancestry outliers (identified using principal components analysis across genetic variants), 58 had technically inadequate scans (spiking artifact, truncated field of view, or signal drop-out or distortion that precluded adequate coregistration), 3 had ventricular size inconsistent with good health in a young person, and 8 lacked a measured genotype at rs6336. The remaining 391 subjects (mean age $23.6 \pm 2.2$ years; range: 20-29 years; 142 males, 249 females) included 88 monozygotic (MZ) twins, 139 dizygotic (DZ) twins or triplets, 41 singleton siblings of other participants included in this study, and 123 individuals who had no twin or singleton sibling included in this study. The Human610-Quad BeadChip (Illumina) was used to analyze DNA according to the manufacturer's protocols (Infinium HD Assay; Super Protocol Guide; Rev. A, May 2008).

All but one of the subjects studied here were included in a previous study of how white matter integrity varied with allele C at Alzheimer's risk gene $C L U$ rs 11136000 . Eight subjects included in that study were excluded from the current one (Braskie et al., 2011). Differences in inclusion were due entirely to the availability of genotypes for the variants in question.

Verbal, Performance, and Full-scale Intelligence Quotient (IQ) standardized scores from the Multidimensional Aptitude Battery (MAB) (Jackson, 1984) were available for 377 subjects. Verbal IQ was composed of subtests of Information, Arithmetic, and Vocabulary. Performance IQ subtests included Spatial and Object Assembly. Full-scale IQ was derived from Verbal and Performance IQ.

All subjects provided written, informed consent. The study conformed to the National Statement on Ethical Conduct in Human Research (2007) issued by the National Health and Medical Research Council of Australia and was approved by the Queensland Institute of Medical Research Human Research Ethics Committee, and by the UCLA Medical Institutional Review Board.

Image acquisition. Each subject underwent MRI scanning ( 4 tesla; Bruker Medspec) that included T1-weighted inversion recovery rapid gradient echo scans $\left(\mathrm{TI} / \mathrm{TR} / \mathrm{TE}=700 / 1500 / 3.35 \mathrm{~ms}\right.$; flip angle $=8^{\circ}$; slice thickness $=0.9$ $\mathrm{mm}, 256 \times 256)$. DTI scans also were acquired using single-shot echo planar imaging with a twice-refocused spin echo sequence to reduce eddy currentinduced distortions $(\mathrm{TR} / \mathrm{TE}=6090 / 91.7 \mathrm{~ms}, 23 \mathrm{~cm}$ FOV, $128 \times 128 ; 552$ $\mathrm{mm}$ axial slices $/ 0 \mathrm{~mm}$ gap; $1.79 \mathrm{~mm} \times 1.79 \mathrm{~mm}$ in-plane resolution). Each DTI scan included 94 diffusion-weighted images $\left(b=1159 \mathrm{~s} / \mathrm{mm}^{2}\right)$ and 11 $b_{0}$ images (i.e., no diffusion sensitization). Gradient directions were evenly distributed on the hemisphere.

DTI preprocessing. Brains were automatically extracted from the T1weighted and diffusion-weighted $b_{0}$ images using ROBEX (Iglesias et al.,
2011). We then manually refined the removal of non-brain matter. All T1-weighted images were linearly aligned ( $9 \mathrm{df}$ ) to a common space. FSL software's (http://fsl.fmrib.ox.ac.uk/fsl/) "eddy_correct" was used to adjust the raw diffusion-weighted images for eddy current distortions. The $b_{0}$ images for each scan were averaged, then linearly aligned and resampled to the T1-weighted image for the same subject (using FSL's linear image registration tool, FLIRT; Jenkinson et al., 2002). They were then elastically registered to the same T1-weighted scan in common space using a mutual information cost function (Leow et al., 2005) to control for echo planar imaging-induced susceptibility artifacts. This provided us with susceptibility-corrected images.

Measures of diffusivity. We used FSL software to create maps of FA and axial and radial diffusivity ( $D_{\mathrm{ax}}$ and $D_{\text {rad }}$, respectively). $D_{\mathrm{ax}}$ is measured as the first tensor eigenvalue $\left(\lambda_{1}\right)$ and represents water diffusion parallel to the axon fiber, when one fiber direction is dominant in the voxel. $D_{\mathrm{rad}}$ is an average of the second and third tensor eigenvalues $\left(\lambda_{2}\right.$ and $\left.\lambda_{3}\right)$ and represents water diffusion perpendicular to the axon fiber. As an additional test, we used mixed-model regression (see Statistical analyses) to evaluate differences in $D_{\mathrm{ax}}$ and $D_{\text {rad }}$ between NTRK1-T carriers and noncarriers to clarify how each might be contributing to the FA differences we found.

Template creation and registration. After performing $b_{0}$ susceptibility correction (see DTI preprocessing), we used FA maps from 32 randomly selected, unrelated subjects (matched for sex) to create a mean deformation target (template brain) (Jahanshad et al., 2010). Each FA map was fluidly and nonlinearly registered (Leporé et al., 2008) to each of the other FA maps. The resulting $N 3 \mathrm{D}$ vector fields for each subject were averaged and applied to the same subject to bring that subject's scan into a space intermediate to all 32 scans. The template brain was an average of these transformed images. This process preserved the image intensities and anatomical features of the template subject. Use of a study-specific FA template rather than a standard template ensures that template scan characteristics are similar to each individual scan, which may improve scan-to-template alignment. In general, we tend to create a template that is as close as possible to the anatomy of the subjects in the study, both in terms of the geometry of the subregions and the mean FA value, which depends on the voxel size of the scan protocol. If these are better matched, scan-to-template registration is generally more accurate, as the deformation algorithm is initialized close to the solution.

Each susceptibility-corrected FA map in the study was registered to the template brain using a 3D elastic warping technique with a mutual information cost function (Leow et al., 2005). FA maps and the template brain were thresholded at 0.25 (excluding contributions from non-white matter). To improve white matter alignment between individual scans and the template brain, the thresholded FA maps were then registered to the thresholded template brain. Images were spatially smoothed with a Gaussian kernel (7 mm FWHM).

Statistical analyses. Family relatedness between each subject and all others was described using a symmetric $N \times N$ kinship matrix. In this matrix, a coefficient of 1 between two different subjects indicated that they were MZ twins; a value of 0.5 indicated that they were $\mathrm{DZ}$ twins or siblings; and a 0 indicated that they were not closely related. Ancestry outliers were removed so no additional modeling was used to account for population genetic structure between families.

Statistical analyses were performed only in voxels for which FA $>0.3$ (likely white matter) in the template brain, reducing partial volume effects. We used mixed-model regression at each of these voxels to estimate how white matter diffusivity measures (FA, $D_{\mathrm{ax}}$, and $D_{\mathrm{rad}}$ ) differed between NTRK1-T carriers and non-carriers while controlling for age, sex, and family relatedness. This analysis was performed using Efficient Mixed-Model Association (EMMA; http://mouse.cs.ucla.edu/emma/) (Kang et al., 2008) within the $\mathrm{R}$ statistical package (version 2.9.2; http://www.r-project.org/).

We compared FA values between NTRK1-T carriers and non-carriers at each voxel, while adjusting for age and sex. To assess the reproducibility of the effects in nonoverlapping samples, we split our full sample in half by generating a list of all subjects sorted by genotype but randomly sorted within genotype group without regard to family membership. The first half of each genotype group was included in sample one and the 
second half was in sample two. The same analysis used on the full sample was performed on each half of the split sample, including adjusting for age, sex, and kinship.

The widely used false discovery rate (FDR) method was used to control for multiple comparisons across all voxels considered (Benjamini and Hochberg, 1995). FDR controls the expected proportion of null results that are incorrectly identified as significant to a set rate if an effect is present (in our case $5 \%$, similar to the standard $p=0.05$ frequently used to define significance). Using FDR, comparisons (voxels) having significance below a critical $p$ value are considered significantly associated with the variable in question, even given the multiple comparisons. Thus, a higher critical $p$ (closer to 0.05 ) represents a stronger association (i.e., more voxels are considered significant after adjustment for multiple comparisons). Actual $p$ values in many of the significant voxels are much lower than the critical $p$ reported. We used EMMA to test the relationship between NTRK1-T and FA using an additive and a dominant model (grouping heterozygotes and T-allele homozygotes), both of which provided significant and very similar results after false discovery rate correction. Because the dominant model performed slightly better at describing the underlying effect and because only two subjects were in the smallest genotype group, the results reported here are using the dominant model unless otherwise stated.

After removing one MZ twin from each pair, a two-tailed Student's $t$ test was used to evaluate whether NTRK1-T carriers and non-carriers were different in age. In the same non-genetically identical subjects, a $\chi^{2}$ test was used to evaluate differences in sex between groups. In a subsample of 377 subjects for whom IQ values were available, we assessed whether Performance IQ, Verbal IQ, or Full-scale IQ was different between NTRK1-T carriers and non-carriers after adjusting for age, sex, and family relatedness. We also evaluated how NTRK1-T related to FA while controlling for age, sex, and IQ in those subjects, again using EMMA for both analyses.

Linkage disequilibrium. Linkage disequilibrium is the non-random association of alleles at two loci. Thus, if a given allele in one singlenucleotide polymorphism (SNP) is associated with brain differences, we would expect that another SNP in moderate linkage disequilibrium with the first might show similar, but weaker, effect. NTRK1 rs6336 is a nonsynonymous coding mutation whose minor allele $\mathrm{T}$ codes for tyrosine, rather than the histidine encoded by the $\mathrm{C}$ allele at position 598 within the kinase domain (van Schijndel et al., 2009). We used the PLINK whole genome association analysis toolset (http://pngu.mgh.harvard.edu/ $\sim$ purcell/plink/) (Purcell et al., 2007) to identify polymorphisms having moderate linkage disequilibrium with $\mathrm{rs} 6336\left(0.20<R^{2}<0.80\right)$. PLINK identified only one SNP ( rs4661063, in an intron of NTRK1) that met this criterion $\left(R^{2}=0.24\right)$. As an additional test to provide support for our main analysis, we used EMMA to evaluate the relationship between rs4661063 and FA, controlling for age, sex, and kinship using additive and dominant models, $7 \mathrm{~mm}$ FWHM spatial smoothing, and evaluating only voxels for which the mean FA $>0.30$ (as with the rs6336 analyses).

Specificity testing. As an additional test to determine the specificity of our NTRK1 results, we used EMMA in the same sample of 391 subjects to evaluate the relationship between FA and genotype for six SNPs that increase the risk for two complex diseases known to be associated with white matter deficits (Peters et al., 2010; Ramli et al., 2010; Thomason and Thompson, 2011). Three of these SNPs were the top SNPs currently associated with schizophrenia (Allen et al., 2008): PRSS16 rs6932590, PGBD1 rs13211507, and NRGN rs12807809, and three were the top SNPs associated with multiple sclerosis (Lill et al., 2011): HLA-DRB1 rs3135388, IL2RA rs2104286, and IL7R rs6897932. As with our NTRK1 analysis, we used a Gaussian smoothing kernel of $7 \mathrm{~mm}$ FWHM; we limited our analysis to voxels for which FA $>0.3$ on the group FA map template. To allow for different possible modes of allelic action, we ran these analyses using the additive and dominant models.

Mean FA for cortical connections. In addition to assessing differences in voxelwise FA between genotypes, we evaluated differences in the mean FA for the tracts connecting cortical regions based on connectivity analysis (Jahanshad et al., 2011). This method uses whole-brain tractography to compute the set of fibers connecting 70 cortical regions of interest, and collects the fibers into sets that connect any pair of regions. With this method, we can compute the mean FA for all fibers that intersect (1) any given cortical region (e.g., the precuneus) or (2) any pair of regions (e.g., precuneus and cuneus). This alternate analysis is based on tractography in individual subjects, and therefore increases the likelihood that the same connections are being compared across subjects, by explicitly identifying their points of origin and their endpoints on a subject-specific basis.

Cortical regions were delineated on each subject's T1-weighted anatomical image as follows. First, non-brain matter was removed from the T1-weighted images using ROBEX (Iglesias et al., 2011), and brain extraction was refined manually. T1-weighted images were linearly aligned to a common space $(1 \mathrm{~mm}$ isotropic voxels; $220 \times 220 \times 220)$ using FSL (with $9 \mathrm{df}$ ). Cortical labels were automatically extracted from all aligned T1-weighted images using FreeSurfer (http://surfer.nmr.mgh.harvard.edu/). For delineation of FreeSurfer cortical regions, see Desikan et al. (2006). Labeled regions in each hemisphere included the banks of the superior temporal sulcus, caudal anterior cingulate cortex, caudal middle frontal gyrus, corpus callosum, cuneus, entorhinal cortex, fusiform gyrus, inferior parietal lobule, inferior temporal gyrus, the isthmus of the cingulate cortex, lateral occipital cortex, lateral orbitofrontal cortex, lingual gyrus, medial orbitofrontal cortex, middle temporal gyrus, parahippocampal gyrus, paracentral lobule, pars opercularis, pars orbitalis, pars triangularis, pericalcarine cortex, postcentral gyrus, posterior cingulate cortex, precentral gyrus, precuneus, rostral anterior cingulate cortex, rostral middle frontal cortex, superior frontal gyrus, superior parietal lobule, superior temporal gyrus, supramarginal gyrus, frontal pole, temporal pole, transverse temporal gyrus, and insular cortex. The labeled cortical models were aligned back to each individual's T1-weighted images and down-sampled using nearest neighbor interpolation (to avoid intermixing of labels) to more closely resemble the diffusion-weighted imaging spatial resolution in the same space. To ensure that the tracts intersected labeled cortical boundaries, labels were all simultaneously dilated with an isotropic box kernel of 5 voxels.

Whole-brain tractography. For tractography, we used the published method by Jahanshad et al. (2011). Briefly, the transformation matrix from the linear alignment of the mean non-diffusion weighted $\left(b_{0}\right)$ image to the T1-weighted volume was applied to each of the 94 diffusion image gradient directions to properly reorient the orientation distribution functions (ODFs). ODFs can describe the directional diffusion of water in regions where multiple fibers cross. At each voxel, we computed ODFs using the normalized and dimensionless ODF estimator, derived for Q-ball imaging (Aganj et al., 2010). We then used these ODFs to perform tractography on the linearly aligned sets of diffusion-weighted volumes. Tracts were derived probabilistically from voxel seeds selected with a prior probability based on the FA value derived from the single-tensor model (Basser and Pierpaoli, 1996). All curves passing through each seed point were scored based on how likely it was that a fiber was present. The Hough transform was used to determine the best fitting curves through each point (Aganj et al., 2011).

The mean FA for the fibers from each cortical region and fibers between each pair of cortical regions was calculated and compared by genotype group, controlling for the effects of sex, age, and kinship. To ensure that only robustly extracted and consistently occurring connections were evaluated, we only compared connections by genotype if those connections had fibers detected in at least $95 \%$ of the study subjects.

Of the 391 scans included in our FA comparisons, 359 were also included in this connectivity evaluation. Subjects excluded were 21 for whom the FreeSurfer cortical segmentation was inadequate, 10 for whom Hough tractography was too sparse and failed to adequately cover the full brain, and 1 for whom the echo planar imaging correction was sufficient for calculating voxelwise FA, but not for tractography.

\section{Results}

At NTRK1 rs6336, 360 subjects were C/C (92.1\%), 29 were C/T (7.4\%), and 2 were $\mathrm{T} / \mathrm{T}(0.5 \%)$ (i.e., $31 \mathrm{~T}$-allele carriers and 360 non-carriers) (Table 1). Similarly, among the $\mathrm{MZ}$ twins, 41 pairs were $\mathrm{C} / \mathrm{C}(93.2 \%), 3$ pairs were $\mathrm{C} / \mathrm{T}(6.8 \%)$, and 0 pairs were $\mathrm{T} / \mathrm{T}$. The minor allele frequency for the $\mathrm{T}$ allele was 0.05 (i.e., $5 \%$ ) in 
Table 1. Demographics for the NTRK1-T carriers versus non-carriers

\begin{tabular}{llc}
\hline Genotypes & T carriers & Tnon-carriers \\
\hline Subjects $(n)$ & 31 & 360 \\
Sex & $16 \mathrm{M}, 15 \mathrm{~F}$ & $126 \mathrm{M}, 234 \mathrm{~F}$ \\
Age $^{a}$ & $23.8 \pm 2.5$ & $23.6 \pm 2.2$ \\
IQ test scores $_{\text {Performance IQ }}{ }^{a}$ & 29 & 348 \\
Verbal IQ $^{a}$ & $119.9 \pm 14.9$ & $113.7 \pm 15.6$ \\
Full-scale IQ $^{a}$ & $114.8 \pm 10.7$ & $111.8 \pm 10.8$ \\
\hline
\end{tabular}

Sex, age, and IQ scores were not significantly different between genotype groups $(p>0.05)$. After removing one twin from each MZ pair, we used a two-tailed $t$ test to evaluate group differences in age and a $\chi^{2}$ test to evaluate group differences in sex. In all subjects, we used multiple regression to evaluate group differences in IQ while controlling for age, sex, and kinship. M, Male; F, female.

${ }^{a}$ Values are mean $\pm S D$.

unrelated subjects in our sample. This is similar to the 0.042 reported previously in $\mathrm{CEPH}$ (Utah residents with ancestry from northern and western Europe, studied by the Centre d'Etude du Polymorphisme Humain, Paris, France; The International HapMap 3 Consortium, 2010) and within the range reported previously (from 0.0179 in a cohort from Norway to 0.0736 in a Swedish cohort; Van Schijndel et al., 2011). The genotype distribution in our sample showed no deviation from the HardyWeinberg equilibrium $\left(\chi^{2}(1)=2.26, p=0.13\right)$.

At NTRK1 rs4661063, 324 subjects were G/G (82.9\%), 64 were $\mathrm{A} / \mathrm{G}(16.4 \%)$, and 3 were A/A (0.8\%). Among the MZ twins, 37 pairs were $\mathrm{G} / \mathrm{G}(82.2 \%), 8$ pairs were $\mathrm{A} / \mathrm{G}(17.8 \%)$, and 0 pairs were A/A. The minor allele frequency for the A allele was $8.8 \%$ in unrelated subjects in our sample. This is the same frequency reported previously in CEPH (Utah residents with ancestry from northern and western Europe; The International HapMap 3 Consortium, 2010). The genotype distribution in our sample did not deviate from the Hardy-Weinberg equilibrium $\left(\chi^{2}(1)=0.0\right.$, $p=1)$.

For our specificity testing, genotypes were as follows: PRSS16 rs6932590 (17 C/C; $135 \mathrm{C} / \mathrm{T} ; 239 \mathrm{~T} / \mathrm{T}$ ), PGBD1 rs13211507 (1 C/C; 58 C/T; 332 T/T), NRGN rs12807809 (11 C/C; 121 C/T; 259 T/T), HLA-DRB1 rs3135388 (8 A/A; 111 A/G; 272 G/G), IL2RA rs2104286 (31 G/G; 172 G/A; 188 A/A), and IL7R rs6897932 (29 T/T; $166 \mathrm{~T} / \mathrm{C} ; 196 \mathrm{C} / \mathrm{C})$.

As shown in Table 1, the NTRK1-T carriers and non-carriers in our sample were not significantly different in age $(t=0.33 ; p=$ $0.74)$ using a two-tailed $t$ test. Sex was not significantly different between carriers and non-carriers using a $\chi^{2}$ test $\left(\chi^{2}(1)=3.03\right.$, $p=0.052$ ), though a strong trend existed toward a greater proportion of males being T-allele carriers. NTRK1-T carriers had significantly higher Performance IQ $(t=2.04 ; p=0.042)$ and Full-scale IQ $(t=2.01 ; p=0.045)$, but not Verbal IQ $(t=1.44$; $p=0.150)$ compared with non-carriers using two-tailed $t$ tests. However, after adjusting for age, sex, and kinship, NTRK1-T carriers and non-carriers did not have significantly different Fullscale IQ $(p=0.16)$, Performance IQ $(p=0.11)$, or Verbal IQ $(p=0.39)$.

We calculated FA in 391 young adults. After controlling for age, sex, and kinship, NTRK1-T carriers had lower FA in frontal, temporal, parietal, occipital and subcortical white matter (multiple-comparisons corrected: critical $p=0.038$, for an FDR controlled at 5\%) (Fig. 1). These regions included the bilateral corona radiata (superior, anterior, and posterior), corticospinal tract, superior and inferior longitudinal fasciculus (SLF and ILF), inferior fronto-occipital fasciculus (IFO), corpus callosum (genu, splenium, body, tapetum, forceps major and minor), cingulum, perithalamic and periventricular white matter, anterior and posterior thalamic radiation (including optic radiations), external capsule, uncinate fasciculus, cerebral peduncles, fornix, and stria terminalis. NTRK1-T was also associated with lower FA broadly throughout the white matter using an additive model (multiple-comparisons corrected: critical $p=0.033$, for an FDR controlled at 5\%). The critical $p$-value was slightly higher using a dominant model, suggesting an underlying dominant effect on the white matter. Even so, only two (unrelated) subjects had a T/T genotype, so it may not be possible to distinguish an additive effect. Since the dominant model had a slightly stronger effect in our sample, our remaining comparisons for rs6336 were performed using that model. No voxels were significantly associated with higher FA in NTRK1-T carriers, after appropriate correction for multiple comparisons.

We next split our full sample into two halves matched for genotype, but randomly assigned to half-sample groups within genotype without regard to family membership. Each group contained $180 \mathrm{C} / \mathrm{C}$ subjects and $1 \mathrm{~T} / \mathrm{T}$ subject. Sample one had 15 $\mathrm{C} / \mathrm{T}$ subjects, while sample two had 14. After controlling for age, sex, and kinship within each subsample, NTRK1-T carriers had significantly lower regional white matter FA compared with noncarriers (sample 1 multiple-comparisons corrected: critical $p=$ 0.012, for an FDR controlled at 5\%; sample 2 multiple-comparisons corrected: critical $p=0.004$, for an FDR controlled at 5\%). Voxels in which NTRK1-T was associated with lower FA in both samples (after FDR correction) were present in the bilateral superior corona radiata (within supramarginal, precentral, and postcentral gyri), ILF, and IFO, right SLF and forceps major, left forceps minor, genu, cingulum, and uncinate fasciculus (Fig. 1).

We again compared FA in NTRK1-T carriers and noncarriers, adjusting for age, sex, full-scale IQ, and kinship in the 377 subjects for whom full-scale IQ was available. FA was still significantly lower in NTRK1-T carriers (multiple-comparisons corrected: critical $p=0.037$, for an FDR controlled at 5\%), suggesting that the variance in FA was accounted for by variance in genotype above and beyond any IQ variance.

We next used EMMA to perform additional tests investigating the nature of our FA differences. Greater $D_{\text {rad }}$ or lower $D_{\text {ax }}$ (see Materials and Methods) may contribute to lower FA. We examined how voxelwise $D_{\mathrm{rad}}$ and $D_{\mathrm{ax}}$ differed between NTRK1-T carriers and non-carriers after adjusting for age, sex, and kinship. $D_{\text {rad }}$ was significantly higher in NTRK1-T carriers than in noncarriers (multiple-comparisons corrected: critical $p=0.031$, for an FDR controlled at 5\%) in many of the same regions where carriers had lower FA (Fig. 2). $D_{\mathrm{ax}}$ was not significantly different between carriers and non-carriers.

Using an additive model, lower FA was significantly associated with rs4661063 allele A (multiple-comparisons corrected: critical $p=0.013$, for an FDR controlled at the conventional 5\% level). Voxels that were significantly associated with FA for both rs4661063 (full sample) and rs6336 (split sample overlap; Fig. 1b) were in the bilateral forceps major and ILF/IFO, right corticospinal tract and SLF, and left forceps minor and cingulum. When we ran this analysis again, covarying for genotype at rs6336, there was no longer a significant effect of rs4661063 on FA, suggesting that the apparent effect of rs4661063 resulted from the stronger rs6336 effect. Allele A at rs4661063 was not significantly associated with FA using a dominant model.

We also used EMMA, controlling for age, sex, and kinship to perform additional testing evaluating the specificity of our results. We examined the relationship between DTI voxelwise FA and top variants with the greatest current evidence of being associated with increased risk for schizophrenia and multiple sclero- 

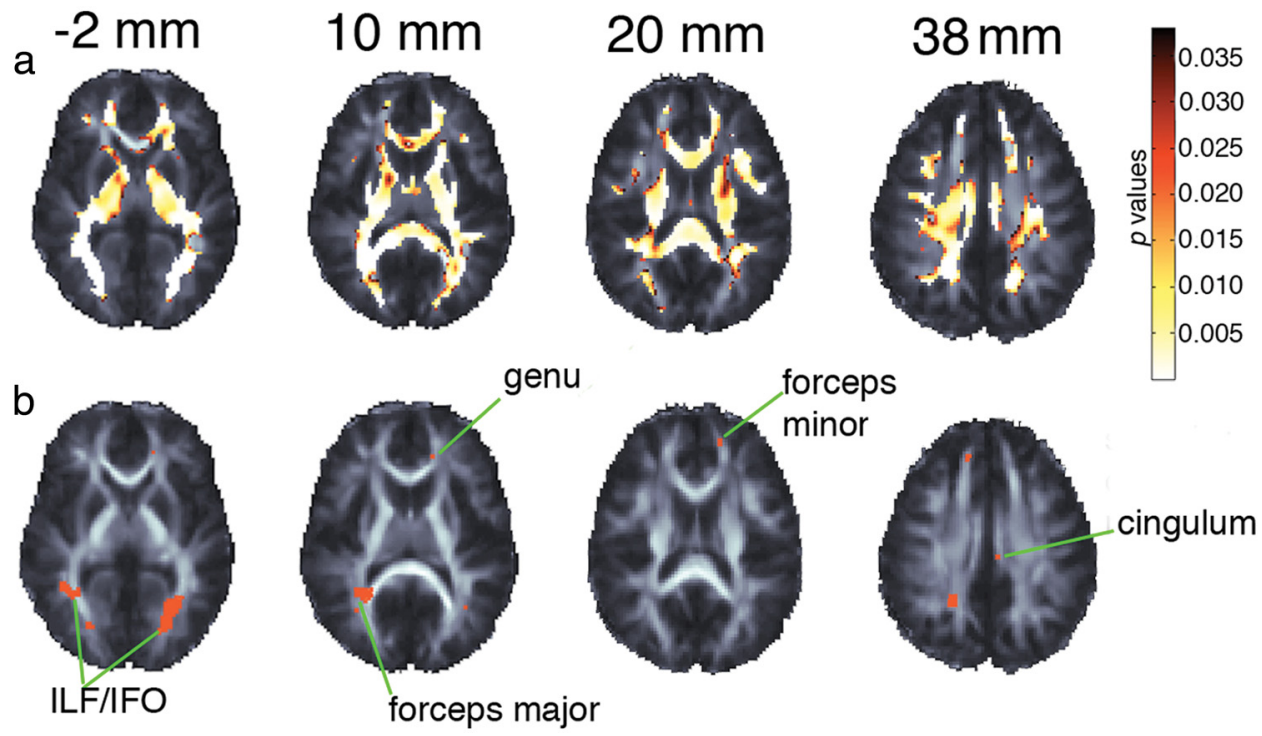

Figure 1. FA association with NTRK1-T displayed on a study-specific FA template. Highlighted areas in $\boldsymbol{a}$ are the $p$-values indicating voxels in which 31 NTRK1-T carriers have significantly lower FA than 360 non-carriers after adjusting for age, sex, and kinship (FDR critical $p$ value $=0.038$ ). In $\boldsymbol{b}$, highlighted voxels are a binary mask indicating in which voxels NTRK1-T carriers showed significantly lower FDR-corrected FA in both halves of the sample displayed in $\boldsymbol{a}$ after adjusting for age, sex, and kinship. Statistical tests were two-tailed. The left brain hemisphere is displayed on the right. Coordinates listed are for the Z-direction in ICBM space.
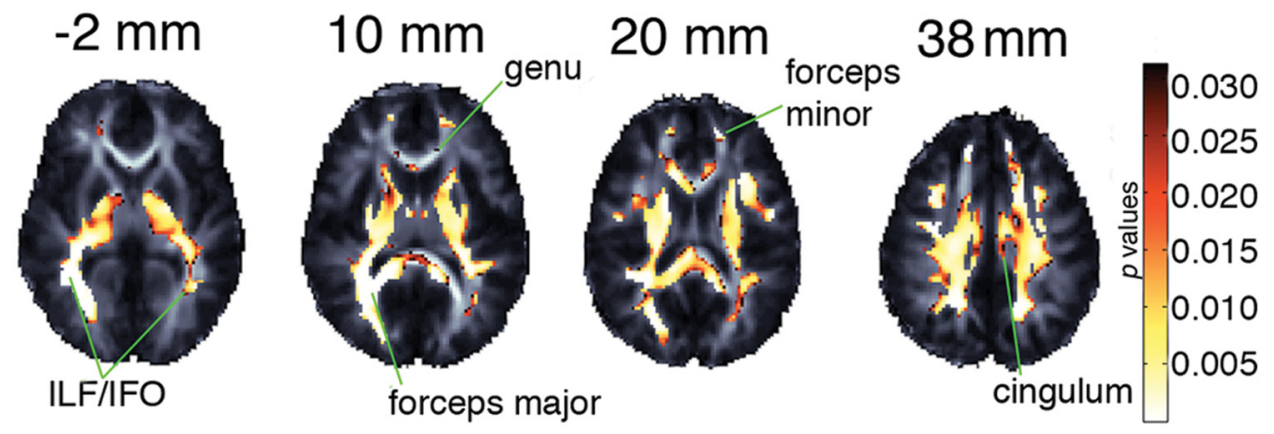

Figure 2. $D_{\text {rad }}$ association with NTRK1-T displayed on a study-specific FA template. Highlighted areas are the $p$-values indicating voxels in which 31 NTRK1-T carriers have significantly higher $D_{\text {rad }}$ than 360 non-carriers after adjusting for age, sex, and kinship ( $F D R$ critical $p$ value $=0.031$ ). The statistical test was two-tailed. The left brain hemisphere is displayed on the right. Coordinates listed are for the Z-direction in ICBM space.

sis. PRSS16 rs6932590, PGBD1 rs13211507, NRGN rs12807809, HLA-DRB1 rs3135388, IL2RA rs2104286, and IL7R rs6897932 were not significantly associated with FA using either an additive or dominant model.

Finally, we examined the extent to which average FA associated with tractography varied with genotype at rs6336. Lower FA was associated with NTRK1-T broadly throughout the brain (Table 2; Fig. 3), including in regions that overlap with the voxelwise FA results in this study.

\section{Discussion}

We found that healthy NTRK1-T carriers had lower FA broadly throughout the brain. Dysfunctions in NGF signaling pathways may contribute to neurodevelopmental deficits, including dysconnectivity (Shoval and Weizman, 2005). NTRK1 is a highaffinity receptor for the neurotrophin, NGF, and is therefore a good candidate for modulating such developmental effects. For instance, NGF has been shown to inhibit central myelination via its receptor, NTRK1, by decreasing the generation and maturation of oligodendrocytes (Chan et al., 2004). In part, this may be because NGF and NTRK1 both help regulate LRR [leucine-rich repeat] and Ig domain-containing Nogo receptor-interacting protein (LINGO-1), which is a strong axonal inhibitor of oligodendrocyte differentiation and myelination (Lee et al., 2007). The relationship we found between NTRK1-T and FA was strong and was replicated in a split sample test, making the variant an interesting target for future studies of development in young adults.

When we analyzed each half of our sample separately, regions in which NTRK1-T showed lower FA in each half-sample included the bilateral superior corona radiata, ILF, and IFO, right SLF and forceps major, and left forceps minor, genu, cingulum, and uncinate fasciculus. The Val allele at rs6265 in the neurotrophin-encoding gene $B D N F$ also showed lower FA in the genu/forceps minor and ILF/IFO previously (Chiang et al., 2011), suggesting that neurotrophin signaling pathways may be especially important to white matter development in these regions.

We identified one SNP in the NTRK1 gene that was in moderate linkage disequilibrium with rs6336. This variant (rs4661063), was significantly associated with FA including in regions significant for 
Table 2. Significant differences between NTRK1-T carriers and non-carriers in mean FA for cortical connections (after FDR correction with critical $p=0.019$ )

\begin{tabular}{|c|c|c|c|c|c|}
\hline \multicolumn{2}{|c|}{ Mean FA is lower in NTRK1-T carriers in fibers that intersect these regions: } & \multicolumn{4}{|c|}{ Mean FA is lower in NTRK1-T carriers in fibers that connect these regions: } \\
\hline & \multicolumn{2}{|r|}{ From } & & To \\
\hline RL & Caudal anterior cingulate cortex & $\mathrm{R}$ & Caudal anterior cingulate cortex & $\mathrm{L}$ & Superior frontal gyrus \\
\hline RL & Rostral anterior cingulate cortex & L & Rostral anterior cingulate cortex & L & Lateral orbitofrontal cortex \\
\hline RL & Caudal middle frontal gyrus & RL & Medial orbitofrontal cortex & ips & Lateral orbitofrontal cortex \\
\hline RL & Lateral orbitofrontal cortex & L & Precentral gyrus & L & Paracentral lobule \\
\hline RL & Medial orbitofrontal cortex & $\mathrm{L}$ & Precentral gyrus & $\mathrm{L}$ & Pars opercularis \\
\hline $\mathrm{R}$ & Paracentral lobule & $\mathrm{L}$ & Precentral gyrus & $\mathrm{L}$ & Posterior cingulate gyrus \\
\hline RL & Pars opercularis & R & Precentral gyrus & $\mathrm{R}$ & Caudal middle frontal gyrus \\
\hline RL & Precentral gyrus & RL & Precentral gyrus & ips & Postcentral gyrus \\
\hline RL & Rostral middle frontal cortex & $\mathrm{RL}$ & Precentral gyrus & ips & Posterior cingulate cortex \\
\hline RL & Superior frontal gyrus & $\mathrm{RL}$ & Insular cortex & $\mathrm{R}$ & Caudal middle frontal gyrus \\
\hline RL & Lateral occipital cortex & $\mathrm{R}$ & Rostral middle frontal cortex & $\mathrm{R}$ & Medial orbitofrontal cortex \\
\hline RL & Lingual gyrus & $\mathrm{R}$ & Rostral middle frontal cortex & $\mathrm{R}$ & Pars opercularis \\
\hline RL & Inferior parietal lobule & $\mathrm{RL}$ & Superior frontal gyrus & ips & Caudal anterior cingulate cortex \\
\hline RL & Postcentral gyrus & $\mathrm{RL}$ & Superior frontal gyrus & ips & Caudal middle frontal gyrus \\
\hline RL & Precuneus & $\mathrm{L}$ & Superior frontal gyrus & L & Lateral orbitofrontal cortex \\
\hline RL & Superior parietal lobule & L & Superior frontal gyrus & L & Rostral anterior cingulate cortex \\
\hline RL & Supramarginal gyrus & L & Superior frontal gyrus & L & Rostral middle frontal cortex \\
\hline $\mathrm{R}$ & Banks of the superior temporal sulcus & $\mathrm{R}$ & Superior frontal gyrus & $\mathrm{R}$ & Precentral gyrus \\
\hline RL & Fusiform gyrus & L & Insular cortex & L & Fusiform gyrus \\
\hline R & Inferior temporal gyrus & L & Insular cortex & L & Lingual gyrus \\
\hline RL & Middle temporal gyrus & $\mathrm{R}$ & Insular cortex & $\mathrm{R}$ & Inferior temporal gyrus \\
\hline \multirow{33}{*}{$\begin{array}{l}\mathrm{ML} \\
\mathrm{RL}\end{array}$} & Superior temporal gyrus & $\mathrm{R}$ & Insular cortex & $\mathrm{R}$ & Pars opercularis \\
\hline & Transverse temporal gyrus & $\mathrm{R}$ & Insular cortex & $\mathrm{R}$ & Superior temporal gyrus \\
\hline & & $\mathrm{R}$ & Lateral occipital cortex & $\mathrm{R}$ & Inferior parietal lobule \\
\hline & & $\mathrm{RL}$ & Lingual gyrus & ips & Fusiform gyrus \\
\hline & & $\mathrm{R}$ & Inferior parietal lobule & $\mathrm{R}$ & Banks of the superior temporal sulcus \\
\hline & & L & Postcentral gyrus & L & Paracentral lobule \\
\hline & & L & Precuneus & $\mathrm{L}$ & Fusiform gyrus \\
\hline & & $\mathrm{L}$ & Precuneus & L & Paracentral gyrus \\
\hline & & $\mathrm{R}$ & Precuneus & $\mathrm{R}$ & Cuneus \\
\hline & & $\mathrm{R}$ & Precuneus & $\mathrm{R}$ & Lateral occipital cortex \\
\hline & & $\mathrm{R}$ & Precuneus & $\mathrm{R}$ & Lingual gyrus \\
\hline & & $\mathrm{L}$ & Superior parietal lobule & L & Paracentral lobule \\
\hline & & L & Superior parietal lobule & $\mathrm{L}$ & Precentral gyrus \\
\hline & & RL & Superior parietal lobule & ips & Postcentral gyrus \\
\hline & & $\mathrm{R}$ & Superior parietal lobule & $\mathrm{R}$ & Cuneus \\
\hline & & $\mathrm{R}$ & Superior parietal lobule & $\mathrm{R}$ & Lateral occipital cortex \\
\hline & & $\mathrm{R}$ & Superior parietal lobule & $\mathrm{R}$ & Precuneus \\
\hline & & $\mathrm{R}$ & Superior parietal lobule & $\mathrm{R}$ & Inferior parietal lobule \\
\hline & & $\mathrm{L}$ & Supramarginal gyrus & $\mathrm{L}$ & Superior temporal gyrus \\
\hline & & RL & Supramarginal gyrus & ips & Postcentral gyrus \\
\hline & & $\mathrm{RL}$ & Supramarginal gyrus & ips & Precentral gyrus \\
\hline & & $\mathrm{R}$ & Inferior temporal gyrus & $\mathrm{R}$ & Fusiform gyrus \\
\hline & & $\mathrm{R}$ & Inferior temporal gyrus & $\mathrm{R}$ & Inferior parietal lobule \\
\hline & & $\mathrm{R}$ & Middle temporal gyrus & $\mathrm{R}$ & Fusiform gyrus \\
\hline & & $\mathrm{R}$ & Middle temporal gyrus & $\mathrm{R}$ & Inferior parietal lobule \\
\hline & & $\mathrm{R}$ & Middle temporal gyrus & $\mathrm{R}$ & Inferior temporal gyrus \\
\hline & & $\mathrm{R}$ & Middle temporal gyrus & $\mathrm{R}$ & Lateral occipital cortex \\
\hline & & $\mathrm{R}$ & Parahippocampal gyrus & $\mathrm{R}$ & Fusiform gyrus \\
\hline & & $\mathrm{R}$ & Superior temporal gyrus & $\mathrm{R}$ & Banks of the superior temporal sulcus \\
\hline & & $\mathrm{R}$ & Superior temporal gyrus & $\mathrm{R}$ & Fusiform gyrus \\
\hline & & $\mathrm{R}$ & Superior temporal gyrus & $\mathrm{R}$ & Inferior temporal gyrus \\
\hline & & $\mathrm{R}$ & Superior temporal gyrus & $\mathrm{R}$ & Middle temporal gyrus \\
\hline & & $\mathrm{R}$ & Transverse temporal gyrus & $\mathrm{R}$ & Superior temporal gyrus \\
\hline
\end{tabular}

R, Right; L, left; ips, ipsilateral.

rs6336 in our split-sample replication. These regions included the ILF/IFO, forceps major and minor, cingulum, SLF, and corticospinal tract. This effect was most likely due to the association of rs4661063 to rs6336, corroborating our discovery of genetic association in those regions.

$D_{\text {rad }}$ (diffusion perpendicular to the axonal fiber) but not $D_{\mathrm{ax}}$ (diffusion parallel to the fiber) was significantly correlated with NTRK1-T in similar regions to those with a DTI-FA effect. Higher $D_{\text {rad }}$ may reflect less myelination, increased permeability of axonal membranes, larger axonal diameters, or less dense axonal packing (Beaulieu, 2002). $D_{\text {rad }}$ may also be influenced by differences in the principal direction of diffusivity for a given voxel (WheelerKingshott and Cercignani, 2009). As there were no significant differences in $D_{\mathrm{ax}}$ between NTRK1-T carriers and non-carriers, the FA effects we found are less likely to relate to axonal damage (WheelerKingshott and Cercignani, 2009). Our results suggest that lower FA in NTRK1-T carriers likely reflects developmental differences rather than specific axonal injury. 
Our voxelwise FA results are largely supported by the differences we found in connectivity-based mean FA throughout the brain. Mean FA results were not expected to match voxelwise FA results exactly; significant differences in mean FA would require a higher degree of invariability of tractography both across subjects and in FA across the full length of a connection to avoid a diluted effect. However, our mean FA results, like our voxelwise results, were significant in the white matter of all brain lobes and in the cingulate region, providing further evidence of the strong effect of NTRK1-T on white matter FA.

Higher FA does not always imply better neuronal function. For instance, some studies found that within a group of schizophrenia patients, hallucinations were associated with higher regional FA (Shergill et al., 2007; Szeszko et al., 2008). However, across neurological diseases and disorders and cognitive domains, lower FA is typically associated with poorer fiber coherence and myelination, and lower cognitive function (Thomason and Thompson, 2011).

Our subjects were aged 20-29. Therefore, myelination of many brain regions was likely still ongoing (Bartzokis et al., 2010; Lebel and Beaulieu, 2011; Lebel et al., 2012). A previous longitudinal study of myelination during development showed that myelin levels increase in individual brain regions with age, peak, and then lose myelin integrity at a generally slower rate than the previous rate of gain (Lebel and Beaulieu, 2011). At any given young adult age, some subjects will have

FA values that may continue to increase in a specific tract, some will have reached their peak with FA levels that have temporarily plateaued, and some will already be showing decreases in FA in any given brain region (Lebel and Beaulieu, 2011). Extent of myelination is dependent on how fast the processes of myelin creation and breakdown are, and their relative balance (Bartzokis et al., 2010). Because our data are cross-sectional, we cannot determine whether the lower FA associated with NTRK1-T relates to generally lower white matter integrity or to delays in myelination that may lead to diminished differences between alleles in more mature adults. However, we found significant correlations between NTRK1-T and FA in the fornix, and strongly in the genu, which on average reach their peak FA before age 20 and at age 21 , respectively (Lebel et al., 2012). Since $74 \%$ of our subjects were older than age 21 , it is relatively unlikely that a delay in myelination related to NTRK1-T fully explains our results. Whether our results relate to less myelination or delayed myelination in NTRK1-T carriers, it remains compelling that young adult carriers of this allele have differences in microstructure relative to the noncarriers of the allele. These differences may result in cognitive or behavioral differences that have yet to be identified and explored.

In a recent meta-analysis, NTRK1-T was associated with a higher probability in Caucasians of developing schizophrenia versus the C allele (van Schijndel et al., 2009), although those results were mixed (Van Schijndel et al., 2011). We found significant effects of NTRK1-T in regions that included the ILF/IFO, cingulum, and genu of the corpus callosum, which most consistently showed lower FA in schizophrenia patients versus controls in a recent meta-analysis (Ellison-Wright and Bullmore, 2009). Brain connectivity deficits are widely considered to be integral to schizophrenia pathogenesis (Bullmore et al., 1997; Shoval and Weizman, 2005). The integrity of white matter tracts connecting brain regions is also under strong genetic control (Chiang et al., 2009). Some schizophrenia risk gene variants may therefore affect white matter integrity. However, as our subjects were all healthy younger adults not selected to have an increased risk for schizophrenia, it is likely that NTRK1-T affects white matter integrity broadly through normal development; many of our regions of lower FA are not specifically associated with schizophrenia, but some of the affected regions are frequently implicated in schizophrenia. Having a first-degree relative with schizophrenia is the largest known risk factor for developing schizophrenia (Mortensen et al., 1999), and all of our regions of effect have also been shown to have lower FA in unaffected family members of schizophrenic patients compared with unrelated controls (Hoptman et al., 2008; Camchong et al., 2009; Clark et al., 2011). Additionally, 
both first-degree relatives of schizophrenic patients and those who display symptoms that place them at ultra-high risk for developing schizophrenia show some DTI abnormalities in the frontal white matter, as we do here, suggesting that such abnormalities may reflect an increased risk for schizophrenia (Peters et al., 2010). It is therefore possible that NTRK1-T contributes to schizophrenia risk via weaker or delayed myelination or altered connectivity in regions vulnerable in the disorder, although other genetic and environmental risk factors would likely also need to be present before schizophrenia onset occurred.

In an overlapping sample of subjects, we previously found that allele C of the Alzheimer's disease risk allele rs11136000 in the $C L U$ gene was associated with lower FA. Strongest $C L U$ effects included some of the same regions that showed lower FA for NTRK1-T carriers in each half of our split sample. Regions having strongest results in both studies were the SLF, ILF and IFO. There is no evidence that the two gene effects share the same mechanism, or that they interact; inheritance of risk variants in these genes is not statistically correlated.

To test the specificity of our current results, we evaluated the relationship between DTI FA and six variants associated with disorders known to affect white matter integrity: schizophrenia and multiple sclerosis. None of these top variants for the two diseases (Allen et al., 2008; Lill et al., 2011) was significantly associated with FA in our sample, suggesting that our findings were specific to NTRK1 rs6336, at least among those variants considered. This specificity analysis supports our inference that effects on fiber integrity are at least somewhat specific to inheriting the NTRK1 risk variant, rather than reflecting disease risk in general.

Our study is limited by the lack of cognitive function measures that demonstrate the impact of NTRK1-T carrier-modulated FA differences. Future investigations of how this variant affects brain structure and function are warranted. Gaining insight into how differences in white matter structure relate to cognition and behavior is an important step in understanding normal development and identifying vulnerabilities to disease. Our results suggest that variation in genes that encode neurotrophins and their receptors may be attractive targets for explaining variability in white matter microstructure in healthy young adults.

\section{References}

Aganj I, Lenglet C, Sapiro G, Yacoub E, Ugurbil K, Harel N (2010) Reconstruction of the orientation distribution function in single- and multipleshell q-ball imaging within constant solid angle. Magn Reson Med 64:554-566.

Aganj I, Lenglet C, Jahanshad N, Yacoub E, Harel N, Thompson PM, Sapiro G (2011) A Hough transform global probabilistic approach to multiplesubject diffusion MRI tractography. Med Image Anal 15:414-425.

Allen NC, Bagade S, McQueen MB, Ioannidis JP, Kavvoura FK, Khoury MJ, Tanzi RE, Bertram L (2008) Systematic meta-analyses and field synopsis of genetic association studies in schizophrenia: the SzGene database. Nat Genet 40:827-834.

Bartzokis G, Lu PH, Tingus K, Mendez MF, Richard A, Peters DG, Oluwadara B, Barrall KA, Finn JP, Villablanca P, Thompson PM, Mintz J (2010) Lifespan trajectory of myelin integrity and maximum motor speed. Neurobiol Aging 31:1554-1562.

Basser PJ, Pierpaoli C (1996) Microstructural and physiological features of tissues elucidated by quantitative-diffusion-tensor MRI. J Magn Reson B 111:209-219.

Beaulieu C (2002) The basis of anisotropic water diffusion in the nervous system - a technical review. NMR Biomed 15:435-455.

Benjamini Y, Hochberg Y (1995) Controlling the false discovery rate-a practical and powerful approach to multiple testing. J R Stat Soc Ser B 57:289-300.

Braskie MN, Jahanshad N, Stein JL, Barysheva M, McMahon KL, de Zubicaray GI, Martin NG, Wright MJ, Ringman JM, Toga AW, Thompson PM
(2011) Common Alzheimer's disease risk variant within the CLU gene affects white matter microstructure in young adults. J Neurosci 31:6764-6770.

Bullmore ET, Frangou S, Murray RM (1997) The dysplastic net hypothesis: an integration of developmental and dysconnectivity theories of schizophrenia. Schizophr Res 28:143-156.

Camchong J, Lim KO, Sponheim SR, Macdonald AW (2009) Frontal white matter integrity as an endophenotype for schizophrenia: diffusion tensor imaging in monozygotic twins and patients' nonpsychotic relatives. Front Hum Neurosci 3:35.

Chan JR, Watkins TA, Cosgaya JM, Zhang C, Chen L, Reichardt LF, Shooter EM, Barres BA (2004) NGF controls axonal receptivity to myelination by Schwann cells or oligodendrocytes. Neuron 43:183-191.

Chiang MC, Barysheva M, Shattuck DW, Lee AD, Madsen SK, Avedissian C, Klunder AD, Toga AW, McMahon KL, de Zubicaray GI, Wright MJ, Srivastava A, Balov N, Thompson PM (2009) Genetics of brain fiber architecture and intellectual performance. J Neurosci 29:2212-2224.

Chiang MC, Barysheva M, Toga AW, Medland SE, Hansell NK, James MR, McMahon KL, de Zubicaray GI, Martin NG, Wright MJ, Thompson PM (2011) BDNF gene effects on brain circuitry replicated in 455 twins. Neuroimage 55:448-454.

Clark KA, Nuechterlein KH, Asarnow RF, Hamilton LS, Phillips OR, Hageman NS, Woods RP, Alger JR, Toga AW, Narr KL (2011) Mean diffusivity and fractional anisotropy as indicators of disease and genetic liability to schizophrenia. J Psychiatr Res 45:980-988.

Desikan RS, Ségonne F, Fischl B, Quinn BT, Dickerson BC, Blacker D, Buckner RL, Dale AM, Maguire RP, Hyman BT, Albert MS, Killiany RJ (2006) An automated labeling system for subdividing the human cerebral cortex on MRI scans into gyral based regions of interest. Neuroimage 31:968-980.

Ellison-Wright I, Bullmore E (2009) Meta-analysis of diffusion tensor imaging studies in schizophrenia. Schizophrenia Res 108:3-10.

Hoptman MJ, Nierenberg J, Bertisch HC, Catalano D, Ardekani BA, Branch CA, Delisi LE (2008) A DTI study of white matter microstructure in individuals at high genetic risk for schizophrenia. Schizophrenia Res 106:115-124.

Iglesias JE, Liu CY, Thompson PM, Tu Z (2011) Robust brain extraction across datasets and comparison with publicly available methods. IEEE Trans Med Imaging 30:1617-1634.

The International HapMap 3 Consortium (2010) Integrating common and rare genetic variation in diverse human populations. Nature 467:52-58.

Jackson DN (1984) MAB, multidimensional aptitude battery: manual. Port Huron, MI: Research Psychologists Press.

Jahanshad N, Lee AD, Barysheva M, McMahon KL, de Zubicaray GI, Martin NG, Wright MJ, Toga AW, Thompson PM (2010) Genetic influences on brain asymmetry: a DTI study of 374 twins and siblings. Neuroimage 52:455-469.

Jahanshad N, Agani I, Lenglet C, Jin Y, Joshi A, Barysheva M, McMahon KL, de Zubicaray GI, Martin NG, Wright MJ, Toga AW, Sapiro G, Thompson PM (2011) High angular resolution diffusion imaging (HARDI) tractography in 234 young adults reveals greater frontal lobe connectivity in women. In: IEEE 8th International Symposium on Biomedical Imaging, pp 939-943.

Jenkinson M, Bannister P, Brady M, Smith S (2002) Improved optimization for the robust and accurate linear registration and motion correction of brain images. Neuroimage 17:825-841.

Kang HM, Zaitlen NA, Wade CM, Kirby A, Heckerman D, Daly MJ, Eskin E (2008) Efficient control of population structure in model organism association mapping. Genetics 178:1709-1723.

Lebel C, Beaulieu C (2011) Longitudinal development of human brain wiring continues from childhood into adulthood. J Neurosci 31:10937-10947.

Lebel C, Gee M, Camicioli R, Wieler M, Martin W, Beaulieu C (2012) Diffusion tensor imaging of white matter tract evolution over the lifespan. Neuroimage 60:340-352.

Lee X, Yang Z, Shao Z, Rosenberg SS, Levesque M, Pepinsky RB, Qiu M, Miller RH, Chan JR, MiS (2007) NGF regulates the expression of axonal LINGO-1 to inhibit oligodendrocyte differentiation and myelination. J Neurosci 27:220-225.

Leow A, Huang SC, Geng A, Becker J, Davis S, Toga A, Thompson P (2005) Inverse consistent mapping in $3 \mathrm{D}$ deformable image registration: its construction and statistical properties. Inf Process Med Imaging 19:493-503.

Leporé N, Chou YY, Lopez OL, Aizenstein HJ, Becker JT, Toga AW, Thompson PM (2008) Fast 3D fluid registration of brain magnetic resonance 
images. In: Medical Imaging 2008: Physiology, Function, and Structure from Medical Images. San Diego: SPIE.

Lill CM, Roehr JT, McQueen MB, Bagade S, Schjeide BM, Zipp F, Bertram L (2011) The MSGene Database. Alzheimer Research Forum http:// www.msgene.org/. Accessed on September 9, 2011.

Maglott D, Ostell J, Pruitt KD, Tatusova T (2005) Entrez Gene: genecentered information at NCBI. Nucleic Acids Res 33:D54-D58.

Mortensen PB, Pedersen CB, Westergaard T, Wohlfahrt J, Ewald H, Mors O, Andersen PK, Melbye M (1999) Effects of family history and place and season of birth on the risk of schizophrenia. N Engl J Med 340:603-608.

Peters BD, Blaas J, de Haan L (2010) Diffusion tensor imaging in the early phase of schizophrenia: what have we learned? J Psychiatr Res 44:993-1004.

Purcell S, Neale B, Todd-Brown K, Thomas L, Ferreira MA, Bender D, Maller J, Sklar P, de Bakker PI, Daly MJ, Sham PC (2007) PLINK: a tool set for whole-genome association and population-based linkage analyses. Am J Hum Genet 81:559-575.

Ramli N, Rahmat K, Azmi K, Chong HT (2010) The past, present and future of imaging in multiple sclerosis. J Clin Neurosci 17:422-427.

Shergill SS, Kanaan RA, Chitnis XA, O’Daly O, Jones DK, Frangou S, Williams SC, Howard RJ, Barker GJ, Murray RM, McGuire P (2007) A diffusion tensor imaging study of fasciculi in schizophrenia. J Psychiatry 164:467-473.
Shoval G, Weizman A (2005) The possible role of neurotrophins in the pathogenesis and therapy of schizophrenia. Eur Neuropsychopharmacol 15:319-329.

Szeszko PR, Robinson DG, Ashtari M, Vogel J, Betensky J, Sevy S, Ardekani BA, Lencz T, Malhotra AK, McCormack J, Miller R, Lim KO, GunduzBruce H, Kane JM, Bilder RM (2008) Clinical and neuropsychological correlates of white matter abnormalities in recent onset schizophrenia. Neuropsychopharmacology 33:976-984.

Thomason ME, Thompson PM (2011) Diffusion imaging, white matter, and psychopathology. Annu Rev Clin Psychol 7:63-85.

van Schijndel JE, van Loo KM, van Zweeden M, Djurovic S, Andreassen OA, Hansen T, Werge T, Kallunki P, Pedersen JT, Martens GJ (2009) Threecohort targeted gene screening reveals a non-synonymous TRKA polymorphism associated with schizophrenia. J Psychiatr Res 43:1195-1199.

Van Schijndel JE, Van Zweeden M, Van Loo KM, Djurovic S, Andreassen OA, Hansen T, Werge T, Nyegaard M, Sørensen KM, Nordentoft M, Mortensen PB, Mors O, Børglum AD, Del-Favero J, Norrback KF, Adolfsson R, De Hert M, Claes S, Cichon S, Rietschel M, et al. (2011) Dual association of a TRKA polymorphism with schizophrenia. Psychiatr Genet 21:125-131.

Wheeler-Kingshott CA, Cercignani M (2009) About "axial" and "radial" diffusivities. Magn Reson Med 61:1255-1260. 